Review Article

\title{
ANALYTICAL METHODS FOR THE DETERMINATION OF AMINOGLYCOSIDES ANTIBIOTICS BY CHROMATROGRAPHIC TECHNIQUE
}

\author{
ISLAM SOFIQUL ${ }^{1 *}$, MURUGAN V. ${ }^{1}$, PREMA KUMARI ${ }^{1}$
}

${ }^{*}$ College of Pharmaceutical Sciences, Dayananda Sagar University, Bangaluru 560078, Karnataka, India Email: sofi59964@gmail.com

Received: 09 Dec 2019, Revised and Accepted: 17 Feb 2020

\begin{abstract}
Aminoglycosides antibiotics are considered to be the antimicrobial agents used frequently in the treatment of human diseases caused by a bacterial infection. Most of the aminoglycosides antibiotics are highly polar in nature and they are lacking the UV absorbing chromophore in the molecules. The present articles accentuate the analytical method associated with the analysis of aminoglycosides molecules. Various chromatographic techniques like liquid chromatography, gas chromatography; mass spectrometry were used for the detection of aminoglycosides antibiotics. However, due to its limitation in the ultraviolet-visible spectrophotometry (UV/Vis) technique, different types of detection techniques like coronacharged aerosol detector (CAD), electrochemical detector (ECD) were used as a most powerful and versatile technique for the demonstration of these molecules in the analytical field. Analytical methods help to ensure the quality of the drug products. This review paper is devoted to providing an overview of the key performance technique used for the application and detection of these aminoglycosides molecules.
\end{abstract}

Keywords: Aminoglycosides antibiotics, Chromophore, Liquid chromatography

(C) 2020 The Authors. Published by Innovare Academic Sciences Pvt Ltd. This is an open access article under the CC BY license (http://creativecommons.org/licenses/by/4.0/) DOI: http://dx.doi.org/10.22159/ijpps.2020v12i4.36583. Journal homepage: https://innovareacademics.in/journals/index.php/ijpps

\section{INTRODUCTION}

Aminoglycosides are a group of highly potent antimicrobial agents used frequently in the treatment of human-caused by both gram-positive and gram-negative bacterial infection. This class of antibiotics also has imperative solicitation in veterinary medicine. Streptomycin is the foremost antibiotics isolated from Streptomyces griseus and it is active against gram-negative bacteria, which were used in clinical studies in 1944, followed by neomycin from Streptomyces fradiae, kanamycin from Streptomyces kanamyceticus, gentamicin from Micromonospora purpurea, sisomicin from Micromonospora inyoensis $[1,2]$. Semisynthetic aminoglycosides like netilmicin from Sisomicin, tobramycin from Streptomyces tenebrarius and amikacin from kanamycin [3]. Aminoglycosides molecules contain aminocyclitol and an amino sugar joined to a ribose unit. They interfere with bacterial protein synthesis by binding irreversible to ribosomes. Aminoglycosides antibiotics have lots of contribution towards the health of human and animals. Most of the aminoglycoside antibiotics are derived by the fermentation process. To improve the safety and efficacy of these classes of molecules various chromatographic technique was used to monitor the purity of the molecule [4]. Chromatographic technique especially high-performance liquid chromatography considered to be used mostly for the analysis of these aminoglycosides.

Due to lack of volatility, absence of chromophore, and hydrophilicity of aminoglycosides, some of the methods applied derivatization technique for improvement of their chromatographic performance. Derivatization techniques with simple chromatographic procedure methods have the advantage of reducing analysis time and lower cost of instruments and maintenance. But these derivatization procedures have shown the disadvantage like lack of stability of the solution. Resemble of the similar molecular structure of these aminoglycosides antibiotics makes the separation of these molecules makes quite critical and major challenging. Some of the detection technique methods like mass spectrometry, gas chromatography was used to analyse these antibiotics. Also there is no definite analytical method that has been reported for the detection of impurities or related compounds present in this aminoglycosides antibiotics [5]. Aminoglycoside antibiotics molecules are polar, resistant to acids, bases, heat and not extensively bound to protein [6]. Although plenty of work has been performed to this various class of compounds, there is still huge potential for further research of this compound.
Chromatographic methods used for the analysis of aminoglycosides

Chromatographic technique used for qualitative use

Aminoglycoside molecules have been analyzed in tissues and urine by various techniques like microbiological, radioenzymatic assay (REA), radioimmunoassay (RIA) method and by paper chromatography. These methods are still extensively used but often lack quantitative or qualitative performance. Some of the biological methods like microbiological assays methods which performed based on agar diffusion of the drug and concentration-dependent growth inhibition (inhibition zone) of the test organism inoculated in the agar. But this assay method requires longer time period (24$72 \mathrm{~h}$ ) for its incubation, after which inhibition of bacterial growth can be measured. Numerous factors like incubation temperature, $\mathrm{pH}$ and depth of the agar on plate and ion concentration of test strain incubation time influence the performance of these methods. Additionally, different agar $\mathrm{pH}$ needs to be used for the analysis of various kinds of aminoglycosides molecules. Although microbiological methods are useful, simple and relatively cheap but looks like they are inaccurate and subject to interferences caused by nonspecific inhibitors or other antimicrobial drugs [7].

RIA methods look more promising as compared to the microbiological assay method. RIA methods are very sensitive and specific, but other aminoglycosides might cause interferences during analysis. Aminoglycosides like gentamicin, tobramycin, amikacin, netilmicin, and sisomicin analysed by using RIA technique. Analysis using an RIA method requires complicated parameter optimization and specialization for the analysis. Selection and preparation of suitable procedure is difficult and time-consuming [8].

\section{Chromatographic technique used for quantitative use}

Chromatographic methods for the analysis of aminoglycoside were needed for qualitative and quantitative determinations. However, due to structural similarity, separations between the aminoglycosides are quite difficult and challenging.

Some of the chromatographic analysis performed by using various chromatographic technique like Gas chromatography, Liquid chromatography, Liquid chromatography with mass spectroscopy (LC MS) etc. were discussed in the various section of this paper. 


\section{Gas chromatography (GC)}

Gas chromatography (GC) is mostly used technique for the analysis of volatile, heat-stable compounds. However, direct analysis of theses aminoglycosides using $\mathrm{GC}$ is quite challenging because of the hydrophilic, basic and non-volatile nature of these aminoglycosides molecules. Derivatization technique was used to improve the chromatographic nature of these types of molecules [9].

Trimethyl silyldiethyl amine (TMSDEA) has been used as a derivatizing agent, for the detection of some class of this aminoglycosides molecules. Derivatizing agent like Trimethyl silyldiethyl amine (TMSDEA) are less sensitive and unstable. Consequently, due to this nature of this agent, it produces nonlinear, poor repeatability and low yield. Freeze drying of samples prior to derivatization need to be used to eliminate variations in sample moisture content and solubility. Sealed sample vials, removal of metal parts from the chromatographic system, and on-column injection have been tried to improve repeatability and quantification. Results obtained from this method were remaining poor $[10,11]$.

The components of Kanamycin A, B, and C have been separated as their trimethylsilyl (TMS) derivatives. The TMS derivatives of neomycin, kanamycin also has been identified by mass spectrometry (MS). Derivatization results in silylation of all amino and hydroxyl groups. Various components of aminoglycosides and its stereoisomers have been separated by GC with derivatization technique [12].

Derivatization procedure using trimethylsilylimidazole (TMSI) for silylation of hydroxyl groups and heptafluorobutyric imidazole (HFBI) for heptafluorobutyrylation of amino groups has been reported.

Preu M, Guyot, Petz M have developed a gas chromatography-mass spectroscopy method for the analysis of aminoglycosides antibiotics using experimental design for the optimization of the derivatization reactions. Here the analytes were derivatized using two-step procedure involving trimethylsilylation of the hydroxyl groups with trimethylsilyimidazole and acylation of the amino group with heptafluro-butyrylimidazole [13].

Mineo H, Kaneko S, Koizumi I reported a gas chromatography with FID detector for the determination of 7 penicillins, 3 tetracycline, 23 antibiotics in meat [14].

Using the derivatization technique, Mayhew and Gorbach detected various aminoglycosides like gentamicin, tobramycin, netilmicin and amikacin in serum. Results are satisfactory in accuracy and precision. TMS-heptafluorobutyryl (HFB) is considered as another suitable derivatizing agent used to analyse the aminoglycosides through GC technique [15].

Stead D discuss about the use of various analytical technique like Xray crystallography, nuclear magnetic resonance (NMR), Mass spectroscopy (MS) for the analysis of aminoglycosides [16].

A. P Topolyan has proposed a method for the derivatization of aminoglycosides antibiotics with Tris (2,6-dimethoxy phenyl) carbenium ion by MS technique to detect the presence of sisomicin, tobramycin molecules [17].

\section{Liquid chromatography (LC)}

High-Performance liquid chromatography (HPLC) is considered as an advance form of chromatographic technique. Different substantial chromatographic parameters like Specificity, Precision, Linearity. Accuracy and Robustness were assessed to check the method performance of the HPLC technique [18]. Due to this it was considered as one of the widely used technologies.

The most significant characteristics of this aminoglycosides molecule are the lack of presence of the chromophore group in their molecular structure. Due to this the analysis of these aminoglycosides compounds was quite challenging.

In HPLC technique, the choice of detector is quite important in order to look at the capability of the elution of all the aminoglycoside components peaks in an appropriate wavelength.
Because of the polar nature of the aminoglycoside molecule different types of C18 analytical column were used along with ion pair buffer to perform the analysis in HPLC UV detector

During the survey of several articles, it was found that the derivatization technique required to analys these aminoglycosides antibiotics with HPLC UV detector.

The most commonly used derivatization reagents are orthophthalaldehyde (OPA) and 1-fluoro-2,4-dinitrobenzene (FDNB) for the analysis of these class of compounds.

Various articles were referred to identify the method used for the analysis were presented below.

- Mustafa S and Devi K reported a method to detect Kenamycin using a mobile phase containing $0.1 \mathrm{M}$ disodium tetraborate $(\mathrm{pH} 9.0)$ and water (25:75) with Phenomenex C18 column under isocratic condition using $205 \mathrm{~nm}$ wavelength [19].

- Hao-Ran J, Xiang-peng Li reported a pre-column derivatization HPLC method for the quantitative and qualitative analysis of Kenamycin. Chromatographic condition was established by using the Kromasil C18 column, Mobile Phase: Methanol: Water (40:60), flow rate $0.5 \mathrm{ml} / \mathrm{min}, \mathrm{UV}$ detection at $390 \mathrm{~nm} \mathrm{[20].}$

- Jin-feng W, Hua-xin reported an HPLC method using Nano quantity analyte detector (NQAD). Chromatographic separation was achieved by using a mobile phase $0.2 \%$ Trifluoroacetic acid: Methanol (80:20) with Agilent SB C18 column [21].

- Kim B, Lee $\mathrm{S}$ and Lee H reported a post-column derivatization liquid chromatographic method for the detection of aminoglycosides using derivatization agent known as phenyl isothiocyanateas. Analytical column Capcell-pak C18, Mobile phase combination of Acetonitrile and $0.1 \%$ TFA at $240 \mathrm{~nm}$ wavelength were used during the analysis [22].

- Kalyani L, Rao C V N reported an RP-HPLC method for the determination of Kenamicin using a combination of Methanol: Acetonitrile: Acetate buffer with pH 5.1 (75:20:0.05 v/v/v) as mobile phase. Waters X Terra column and $212 \mathrm{~nm}$ were used a wavelength nanometer during the analysis [23].

- Korany M, Haggag R reported a novel liquid chromatographic technique method using pre-column derivatization reaction to determine amikacin. The separation was achieved by Spherisorb C18 ODS column using Mobile phase composed of Acetonitrile: $0.1 \mathrm{M}$ Sodium acetate buffer ( $\mathrm{pH}$ 5.0, 25:75 v/v). Detection was carried out at $330 \mathrm{~nm}$ [24].

- Dan H, Yang L reported a post-column derivatization method for the determination of amikacin using Waters SunFire C18 column. Detection was carried out 360 and $440 \mathrm{~nm}$ [25].

- Vimal D proposed an RP HPLC method for the estimation of amikacin. The separation was achieved by C18 column and Acetonitrile: water $(10: 90 \mathrm{v} / \mathrm{v})$ as mobile phase. Detection was carried out at $212 \mathrm{~nm}$ [26].

- Feng C H, Lin S developed a simple and sensitive liquid chromatographic method for the determination of amikacin in human plasma. The amikacin is derivatized with 1-naphthyl isothiocyanate (NITC) and it was analysed by HPLC on a LiChroCART RP-Cls column with water-acetonitrile $(57: 43, \mathrm{v} / \mathrm{v})$ as mobile phase and detection carried out at $230 \mathrm{~nm}$ [27].

- Chauhan B, Jalalpure S developed an ultra-high performance liquid chromatography (UHPLC) method for the determination of amikacin sulfate in human serum using derivatization with FMOCCl and glycine. Chromatographic condition was achieved by using mobile phase composed of Acetonitrile: water in the ratio 70:30 (v/v) and Shim-Pack XR-ODS III, Shimadzu) C18 column. Fluorimetric detection at excitation and emission wavelength of 265 $\mathrm{nm}$ and $315 \mathrm{~nm}$, respectively was used for the proposed chromatographic method [28].

- Kim M, Liu Y developed a tandem mass spectroscopy method for the detection of amikacin using a mobile phase composed of $\mathrm{MeOH} / 10$ mmol $\mathrm{NH}_{4} \mathrm{OAc}(\mathrm{pH}$ 4.0)/Heptafluorobutyric acid 
(5/95/0.2) and methanol. Betasil phenyl column $(100 \times 2.1 \mathrm{~mm}$, $5 \mu \mathrm{m}$ ) was used during the analysis [29].

- Li D, He S presented a rapid and sensitive high-performance liquid chromatography method for the determination of amikacin in water samples with solid phase extraction and pre-column derivatization. Solution were derivatized by using 4-chloro 3,5 dinitrobenzotrifluroride in presence of trimethylamine at $70{ }^{\circ} \mathrm{C}$. Kromasil ODS C 18 column was used during the analysis [30].

- Chuong M C, Chin J recommended a HPLC method for the assay of

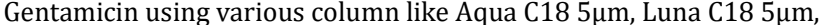
Nucleosil C18 $5 \mu \mathrm{m}$. Mobile phase was prepared by mixing methanol, water, and glacial acetic acid (70:25:5 v/v/v) with Sodium 1heptanesulfonate. Derivatization procedure was performed by using ortho-phthaladehyde (OPA) solution. Detection wavelength was carried out at $330 \mathrm{~nm}$ [31].

- Adams E, Vaerenbergh G V, Roets E reported a liquid chromatographic method for the analysis of amikacin with pulsed electrochemical detection [32].

- Oguri S, Miki Y reported a selective and reproducible high performance capillary electrophoretic (HPCE) method for the quantification of amikacin in human plasma. This method involves ultrafiltration of plasma before derivatization with the fluorescence derivatizing reagents 1-methoxy-caronylindolizine-3.5 dicarbaldehyde at room temperature [33].

- Ovalles J F, Brunetto M R, Gallignani M proposed a simple and sensitive RP HPLC method for the determination of amikacin (AMK) by using derivatization technique. This method is based on the precolumn derivatization of AMK with 6-aminoquinolyl-Nhydroxysuccinimidyl carbamate (AQC). Detection was performed by UV absorption instead of fluorescence [34].

- Galanakis E G, Megoulas N C, Soluch P reported a novel method for the direct determination of the aminoglycosides antibiotics (amikacin, kanamycin) based on the reverse phase liquid chromatographic (LC) with ELSD detector [35].

- Nicoli S, Santi $P$ reported a simple technique for the determination of amikacin by using HPLC UV technique. UV detection was carried out $365 \mathrm{~nm}$ [36].

- Zawilla N H, Li B, Hoogmatens J reported an improved reversedphase liquid chromatographic method with ECD for the analysis of amikacin. Proposed method was performed by using Discovery column [37].

- Serrano M J, Silva M reported a simple and sensitive method for the quantification of amikacin in the urine sample by using HPLC with chemiluminescence detection [38].

- Brajnoski G, Hoogmartens J, Allegaert K proposed a sensitive method for the determination of amikacin by using electrochemical pulse detection [39].

- Zhu Yu C, Zhao He Y reported an analytical method for the determination of kanamycin $\mathrm{A}$, amikacin and tobramycin residues in milk by capillary zone electrophoresis with post column derivatization and laser-induced fluorescence detection [40].

- Laki M, Hajdu M developed a new, fast high-performance liquid chromatographic-UV method for quantitative analysis of gentamicin carrier samples drawn in drug release studies. The mobile phase consisted of methanol-water-acetate buffer $(0.02 \mathrm{M}$ ammonium acetate solution, adjusted with ammonia to $\mathrm{pH}=9$ ), a reverse phase, Zorbax Rx-C18 column has been used during the analysis [41].

- Kuehl P, De S developed a stability-indicating HPLC assay method with UV detection for the simultaneous quantification of Gentamicin Sulfate and L-leucine from NanoGENT dry powder for inhalation. Mobile phase was prepared by mixing methanol, water, and glacial acetic acid (70:25:5) with Sodium 1-heptanesulfonate. Derivatization procedure was performed by using orthopthaladehyde (OPA) solution. Detection wavelength was carried out at $330 \mathrm{~nm}$ during the analysis [42].
- Joseph A, Rustum A developed a RP-HPLC method for the determination of gentamicin sulfate using pentafluorophenyl column and a charged aerosol detector. Mobile phase comprising of (A) heptafluorobutyric acid: water: acetonitrile $(0.025: 95: 5, \mathrm{v} / \mathrm{v} / \mathrm{v})$ and (B) trifluoroacetic acid: water: acetonitrile (1:95:5, v/v/v) was used during the study [43].

- Isoherranen $\mathrm{N}$, Soback S developed a method to determine gentamicin and its components using derivatization technique with UV detector. 1-fluoro-2,4-dinitrobenzene was used as derivatization agents. Symmetry TM C18 reversed-phase column was used during the study [44].

- Meicheng Y, Zhen L developed a liquid chromatographic method using pre column derivatization for the content of gentamicin sulfate and neomycin sulphate. 9-fluorenylmethyl chloroformate (FMOC-Cl) was used for derivatization during the analysis [45].

- Plozza T, Trenerry V C reported a robust method to confirm and quantify the levels of dihydrostreptomycin, streptomycin, apramycin, neomycin and gentamicin (C1, C2 and C1a) present in animal tissue using liquid chromatography-tandem mass spectrometry. The compounds were separated using C18 column and mobile phase consisting of a mixture of acetonitrile, water and $50 \mathrm{mmol}$ heptafluorobutyric acids [46].

- Caudron E, Bagriche S. developed a simple HPLC method for the determination of gentamicin sulfate and colistin sulfate by ion pairing reverse phase chromatography at UV detection $215 \mathrm{~nm}$. Separation was achieved by using Waters X Terra C 18 column. Combination of Acetonitrile: Water was used as mobile phase [47].

- Hussain A, developed a simple high performance liquid chromatographic technique for the estimation of Streptomycin. It was achieved by using intersil ODS-3 C-18 column and detected carried out by UV-Visible Detector at $240 \mathrm{~nm}$. A gradient combination of a mixture of Methanol and Buffer was used as a mobile phase [48].

- T J Whall developed an isocratic high performance liquid chromatographic method for the determination of streptomycin and dihydrostreptomycin. The method employs a microparticulate reversed-phase ( $\mu$ Bondapak C18 and LiChrosorb RP-18) column and a mobile phase composed of $0.02 \mathrm{M}$ sodium hexane sulfonate and $0.025 \mathrm{M}$ tribasic sodium phosphate in acetonitrile-water $(8: 92, \mathrm{v} / \mathrm{v})$ at $\mathrm{pH} 6.0$ with detection by ultraviolet absorbance at $195 \mathrm{~nm} \mathrm{[49].}$

- Bruijnsvoort V M, Ottink M J, Jonker M K developed a LC-MS/MS method for the determination of streptomycin (STR) and its derivative dihydrostreptomycin. Proposed method was achieved by using Analytical Alltima C 18 column [50].

- Edder P, Cominoli A, Corvi C reported a simple and reliable procedure for the analysis of streptomycin by using $\beta$ napthoquinone 4-sulfonate as post derivatization and fluorescence detection [51].

- Holzgrabe U, Nap J C, Kunz N proposed a method to control the impurities in Streptomycin sulfate by HPLC coupled with mass detection and corona charged aerosol detection. This was performed by using the Supelcosil ABZ alkylamide column [52].

- Adams E, Rafiee M, Roets E proposed a liquid chromatographic method for the analysis of streptomycin sulfate. Proposed method was achieved by using analytical column Supelcosil LC-ABZ column [53].

- Ashraf S, Ahmad Z R developed a simple and sensitive ultra-high performance liquid chromatographic method (UPLC) with electron spray ionization (ESI) tandem mass spectrometry. Separation of both the analytes was carried out by using BEH Hillic column and triple quadruple mass spectrometer in positive ESI mode [54].

- Ruckmani K, Shaikh Z developed a novel method for the determination of Tobramycin using UV detector. An isocratic mobile phase consists of buffer $0.05 \mathrm{M}$ diammonium hydrogen phosphate, $\mathrm{pH}$ adjusted to 10.0 using tetramethylammonium hydroxide. Analysis was carried out by using Purosphere RP column. The detection was carried out using variable wavelength UV-Vis detector set at $210 \mathrm{~nm}$ [55]. 
- Russ H, Mecleary developed a HPLC method for the determination of tobramycin in ophthalmic suspension. Proposed method was achieved by using chromatographic parameters include a mobile phase of acetonitrile/buffer (55/45; v/v) and a Nova-Pak C18 column, maintained under ambient conditions. The wavelength of detection was set at $365 \mathrm{~nm}$ [56].

- Zhu L, Wang J developed simple and direct method for the detection of Tobramycin using refractive index (RI) detector. ZORBAX SB-C18 column used was during analysis [57].

- Clarot I, Paris S I developed a simple HPLC method with evaporative light scattering detection for the detection of Tobramycin. Chromatographic separation was carried out in gradient mode using a Zorbax SB C18 column with mobile phase's combination of acetonitrile and water with trifluoroacetic [58].

- Kubo H, Kobayashi Y, Nishikawa T proposed a simple and accurate liquid chromatographic method for the determination of kanamycin and dibekacin in serum. The determination of kanamycin and dibekacin was performed by a combination of reverse-phase, ion-pair chromatography, post column derivatization with orthophthalaldehyde, and fluorescence detection [59].

- Manyanga V, Elkady E have proposed a reversed phase liquid chromatographic method with pulsed electrochemical detection for tobramycin in bulk and pharmaceutical formulation. Chromatographic condition was achieved using a Discovery C18 RP column with a mobile phase, containing sodium sulfate $(35 \mathrm{~g} / \mathrm{l})$, sodium octanesulphonic acid (1 g/l), tetrahydrofuran $(14 \mathrm{ml} / \mathrm{l})$ and $0.2 \mathrm{M}$ phosphate buffer $\mathrm{pH} 3.0$ [60].

- Mashat M, Chrystn H proposed a reversed-phase liquid chromatography method involving pre-column derivatisation with fluorescein isothiocyanate for determination of tobramycin in urine samples. The chromatographic separation was carried out on a Phenomenex Luna C18 column at ambient temperature using mobile phase of acetonitrile-methanol-glacial acetic acid-water (420:60:5:515, v/v/v/v). The tobramycin-FITC derivative was monitored by fluorescent detection at an excitation wavelength 490 $\mathrm{nm}$ and emission wavelength $518 \mathrm{~nm}$ [61].

- Huang L, Haagensen JAJ proposed LC-MS/MS method for the determination of Tobramycin in $\mathrm{M}_{9}$ media. Method performance was achieved by using a PFP column $(2.0 \times 50 \mathrm{~mm}, 3 \mu \mathrm{m})$ eluted with water containing $20 \mathrm{mmol}$ ammonium formate and $0.14 \%$ trifluoroacetic acid and acetonitrile containing $0.1 \%$ trifluoroacetic acid in a gradient mode [62].

\section{DATA SOURCE}

English language article published from 1980 to 2019 were identified through searches of the Pistoia Alliance database, science direct data base, Analytics, Reference standard data base various bibliographies using the key word like Aminoglycosides, chromatography, names of aminoglycosides molecule, liquid chromatography technique. The search include various chromatographic condition uses to analyse the aminoglycoside molecules through various research and review articles. Search dates February 2019 to November 2019

\section{CONCLUSION}

This article provides knowledge for the analysis of aminoglycoside molecule by liquid chromatographic technique. The wider use of this class of compounds requires suitable methods for their detection and use in routine analysis. The proposed methods must be accurate, sensitive, and robust against interferences. However, the chemical features of aminoglycoside molecules such as polarity, solubility, lack of volatility, and lack of chromophore make method development difficult and challenging.

Selection of derivatizing agents and chromatographic techniques plays a substantial role on the separation and selectivity of the method. Developed method need to validated as per the regulatory guideline and it is utilized to ensure that quality is built to support drug development process.

\section{FUNDING}

Nil

\section{AUTHORS CONTRIBUTIONS}

All the author has contributed equally.

\section{CONFLICT OF INTERESTS}

\section{Declared none}

\section{REFERENCES}

1. MJ Weinstein, GM Luedemann, EM Oden. Gentamicin, a new broad-spectrum antibiotic complex. Antimicrobial agents. Chemother (Bethesda) 1963;161:1-7.

2. H Umezawa, IR Hooper. Eds. Aminoglycosides antibiotics; 1982;6:1-368.

3. Edson RS, Terrell CL. The aminoglycosides: streptomycin, kanamycin, gentamicin, tobramycin, amikacin, netilmicin, and sisomicin. Mayo Clin Proc 1987;62:916-20.

4. Goodman and Gilman the pharmacological basis of therapeutics. $13^{\text {th }}$ edition; 2017.

5. Grahek R, Kralji L. Identification of gentamicin impurities by liquid chromatography tandem mass spectrometry. J Pham Bio Anal 2009;50:1037-43.

6. Salam Abdul I, Amoud A, Clark JB. Determination of gentamicin in urine samples after inhalation by reversed-phase highperformance liquid chromatography using pre-column derivatization with o-phthalaldehyde. J Chromatogr B Analyt Technol Biomed Life Sci 2002;769:89-95.

7. DA Bruckner, JA Hindler. WJ Martin. Comparison of radioimmunoassay with a new immunofluorescent method (FIAX) for measuring tobramycin in serum. Antimicrob Agents Chemother 1982;21:107-9.

8. P Stevens, LS Young. Radioimmunoassay of amikacin and comparison with a microassay. J Anti 1976;29:829-32.

9. JW Mayhew, L Gorbach. Gas-liquid chromatographic method for the assay of aminoglycoside antibiotics in serum. J Chromatogr 1978;151:133-46.

10. M Ali. Advanced gas chromatography-progress in agricultural, biomedical and industrial applications; 2012.

11. VK Bajpai, N Kim, K Kim. Chemical derivatization of pharmaceutical samples prior to gas-chromatography and mass-spectrometry analysis bang. J Pharm 2016;11:852-5.

12. A Adegoke. An overview of applications of pre-column derivatization reactions for the liquid chromatographic analysis of pharmaceuticals and other compounds. Afr J Pure Appl Chem 2012;6:129-40.

13. Preu M, Guyot D, Petz M. Development of a gas chromatography-mass spectrometry method for the analysis of aminoglycoside antibiotics using experimental design for the optimisation of the derivatization reactions. J Chromatogr A 1998;818:95-108.

14. Mineo H, Kaneko S, Koizumi I. An analytical study of antibacterial residues in meat: The simultaneous determination of 23 antibiotics and 13 drugs using gas chromatography. Human Toxicol 1992;34:393-7.

15. Mayhew JW, Gorbach SL. Assay of gentamicin and tobramycin in sera of patients by gas-liquid chromatography. Antimicrob Agents Chemother 1978;14:851-5.

16. Stead D. Current methodologies for the analysis of aminoglycosides. J Bio Sci Appl 2000;747:69-93.

17. P Topolyan. Derivatization of aminoglycoside antibiotics with tris (2,6-dimethoxyphenyl) carbenium Ion. Acta Nat 2016;8:129-35.

18. Shivani S, Swapnil G, Kalindi C. A review on analytical method development and validation. Int J Appl Pharm A 2018;10:1-8.

19. Mustafa S, Devi V. Liquid chromatographic assay for the analysis of kanamycin sulphate nanoparticles in rat after intramuscular administration: application to a pharmacokinetic study. J Appl Pharm Sci 2016;6:57-66.

20. Hao Ran J, Xiang Peng Li. Studies of kanamycin sulfate determination method by HPLC with pre-column derivatization. Chinese J Ana Chem 2007.

21. Jin Feng W, Hua Xin. Chinese J Anal Chem 2014;34:644-8. 
22. Kim BH, Lee SC, Lee HJ. Reversed-phase liquid chromatographic method for the analysis of aminoglycoside antibiotics using pre-column derivatization with phenylisocyanate. Biomed Chromatogr 2003;17:396-403.

23. Kalyani L, Rao CVN. Stability indicating RP-HPLC method development and validation of cefepime and amikacin in pure and pharmaceutical dosage forms. Braz J Pharm Sci 2018;54:1-9.

24. Korany M, Haggag R. Liquid chromatographic determination of amikacin sulphate after pre-column derivatization. J Chromatogr Sci 2014;52:837-47.

25. Dan H, Yang L. Determination of amikacin sulfate injection by HPLC with post-column derivatization. Chinese J Anal Chem 2009:29;1025-6.

26. Vimal D. Development and validation of rp-hplc method for simultaneous estimation of cefepime hydrochloride and amikacin sulphate in injection dosage form. J Pharm Sci Bio Sci Res 2012;2:138-43.

27. Feng CH, Lin S. Trace analysis of amikacin in human plasma by high-performance liquid chromatography. Chromatographia 2001;53:213-7.

28. Chauhan B, Jalalpure S. Analysis of amikacin in human serum by UHPLC with fluorescence detector using chloro-formate reagent with glycine. Pharm Methods 2016;7:99-103.

29. Kim M, Liu Y, Kcas Kamsas. A rapid and sensitive LC-MS/MS assay for the quantitation of deacetyl mycoepoxydiene in rat plasma with application to preclinical pharmacokinetics studies. J Chromatogr B: Anal Technol Biomed Life Sci 2012;880:1-5.

30. Li D, He S. Development and validation of an HPLC method for determination of Amikacin in water samples by solid phase extraction and pre-column derivatization. Bull Environ Contam Toxicol 2014;93:47-52.

31. C Chuong, J Chin. High performance liquid chromatography of gentamicin sulfate reference standard and injections. Int J Pharm Anal 2013;4:25-9.

32. Adams E, Vaerenbergh GV, Roets E. Determination of amikacin by liquid chromatography with pulsed electrochemical detection. J Chromatogr A 1998;819:93-7.

33. Oguri S, Miki Y. Determination of amikacin in human plasma by high-performance capillary electrophoresis with fluorescence detection. J Chromatogr B 1996;686:205-10.

34. Ovalles JF, Brunetto MR, Gallignani M. A new method for the analysis of amikacin using 6-aminoquinolyl-Nhydroxysuccinimidyl carbamate (AQC) derivatization and highperformance liquid chromatography with UV-detection. J Pharm Anal 2005;39:294-8.

35. Galanakis EG, Megoulas NC, Soluch P. Development and validation of a novel LC non-derivatization method for the determination of amikacin in pharmaceuticals based on evaporative light scattering detection. J Pharm Bio Anal 2006;40:1114-20.

36. Nicoli S, Santi P. Assay of amikacin in the skin by highperformance liquid chromatography. J Pharm Biomed Anal 2006;41:994-7.

37. Zawilla $\mathrm{NH}, \mathrm{Li} \mathrm{B}$, Hoogmatens J. Improved reversed-phase liquid chromatographic method combined with pulsed electrochemical detection for the analysis of amikacin J Pharm Biomed Anal 2007;43:168-73.

38. Serrano MJ, Silva M. Determination of amikacin in body fluid by high-performance liquid-chromatography with chemiluminescence detection. J Chromatogr B 2006;843:20-4.

39. Brajnoski G, Hoogmartens J, Allegaert K. Determination of amikacin in cerebrospinal fluid by high-performance liquid chromatography with pulsed electrochemical detection. J Chromatogr 2008;867:149-52.

40. Zhu Yu C, Zhao He Y. Determination of kanamycin a, amikacin and tobramycin residues in milk by capillary zone electrophoresis with post-column derivatization and laserinduced fluorescence detection. J Chromatogr 2009;877:333-8.

41. Laki M, Hajdu M. Determination of gentamicin released from orthopedic carrier system by a novel HPLC Method. J Chromatogr Sci 2011;49:177-81.

42. Kuehl P, De S. Development and validation of an HPLC assay for dual detection of gentamicin sulfate and leucine from a novel dry powder for inhalation. J Anal Bioanal Tech 2012;3:1-4.
43. Joseph A, Rustum A. Development and validation of a RP-HPLC method for the determination of gentamicin sulfate and its related substances in a pharmaceutical cream using a short pentafluorophenyl column and a charged aerosol detector. J Pharm Biomed Anal 2010;51:521-31.

44. Isoherranen N, Soback S. Determination of gentamicins C1, $\mathrm{C}(1 \mathrm{a})$, and $\mathrm{C} 2$ in plasma and urine by HPLC. Clin Chem 2000;46:837-42.

45. Meicheng Y, Zhen L. HPLC method using pre-column derivatization for determination of the content of gentamicin sulfate and neomycin sulfate. Chinese J Clin Pharm 2004-05.

46. Plozza T, Trenerry VC. The confirmation and quantification of selected aminoglycoside residues in animal tissue and bovine milk by liquid chromatography tandem mass spectrometry. Int Food Res J 2011;18:1077-84.

47. Caudron E, Bagriche S. Simultaneous quantification of gentamicin and colistin sulfate in pharmaceuticals using ionpairing and polarity gradient chromatography with low-uv detection. Chromatrogrphia 2013;76:744-55.

48. Hussain A. Simple and rapid method on high performance liquid chromatography (HPLC) for estimation of streptomycin sulphate. World Appl Sci J 2012;19:645-9.

49. TJ Whall. Determination of streptomycin sulfate and dihydrostreptomycin sulfate by high-performance liquid chromatography. J Chromatogr A 1981;219:89-100.

50. Bruijnsvoort VM, Ottink MJ, Jonker MK. Determination of streptomycin and dihydrostreptomycin in milk and honey by liquid chromatography with tandem mass spectrometry. J Chromatogr A 2004;1058:137-42.

51. Edder P, Cominoli A, Corvi C. Determination of streptomycin residues in food by solid-phase extraction and liquid chromatography with post-column derivatization and fluorometric detection. J Chromatogr A 1999;830:345-51.

52. Holzgrabe U, Nap JC, Kunz N. Identification and control of impurities in streptomycin sulfate by high-performance liquid chromatography coupled with mass detection and corona charged-aerosol detection. J Pharm Biomed Anal 2011;56:271-9.

53. Adams E, Rafiee M, Roets. Liquid chromatographic analysis of streptomycin sulfate. J Pharm Biomed Anal 2000;24:219-26.

54. Ashraf S, Ahmad ZR. Development and validation of an UPLCESI-MS/MS analytical method for the determination of streptomycin and dihydrostreptomycin residues in honey. Bio Pharm J 2017;10:1983-92.

55. Ruckmani K, Shaikh Z. A simple and rapid high-performance liquid chromatographic method for determining tobramycin in pharmaceutical formulations by direct UV detection. Pharm Method 2011;2:117-23.

56. Russ H, Mecleary. Development and validation of a stabilityindicating HPLC method for the determination of tobramycin and its related substances in an ophthalmic suspension. J Liq Chromatogr Relat Technol 1998;21:2165-81.

57. Zhu L, Wang J. Fast determination of tobramycin by reversedphase ion pair high performance liquid chromatography with a refractive index detector. Front Chem Sci Eng 2013;7:322-8.

58. Clarot I, Paris IS, Chaminade. Simultaneous quantification of tobramycin and colistin sulfate by HPLC with evaporative light scattering detection. J Pharm Bio Sci 2009;50:64-7.

59. Kubo H, Kobayashi Y, Nishikawa T. Rapid method for determination of kanamycin and dibekacin in serum by use of high-pressure liquid chromatography. Antimicrob Agents Chemother 1985;28:521-3.

60. Manyanga V, Elkady E, Hoogmartenus J. Improved reversed phase liquid chromatographic method with pulsed electrochemical detection for tobramycin in bulk and pharmaceutical formulation. J Pharm Anal 2013;3:161-7.

61. Mashat M, Chrystn H, Clark BJ. Development and validation of HPLC method for the determination of tobramycin in urine samples post-inhalation using pre-column derivatisation with fluorescein isothiocyanate. J Pharm Bio Sci 2008;869:59-66.

62. Huang L, Haagensen JAJ. Determination of tobramycin in M9 medium by LC-MS/MS: signal enhancement by trichloroacetic acid. J Anal Methods Chem 2018:1-8. https://doi.org/ $10.1155 / 2018 / 7965124$. 